\title{
The Dependence between Urinary Levels of Angiogenesis Factors, 8-Iso-prostaglandin F2 $\alpha, \gamma$-Synuclein, and Interleukin-13 in Patients with Bladder Cancer: A Pilot Study
}

\author{
Beata Szymańska (D, ${ }^{1}$ Ewa Sawicka, ${ }^{1}$ Michał Matuszewski, ${ }^{2}$ Janusz Dembowski, ${ }^{2}$ \\ and Agnieszka Piwowar ${ }^{1}$ \\ ${ }^{1}$ Department of Toxicology, Faculty of Pharmacy, Wroclaw Medical University, Wroclaw, Poland \\ ${ }^{2}$ Department of Urology and Oncological Urology, Faculty of Medicine, Wroclaw Medical University, Wroclaw, Poland \\ Correspondence should be addressed to Beata Szymańska; beata.szymanska@umed.wroc.pl
}

Received 19 May 2020; Revised 9 November 2020; Accepted 21 November 2020; Published 2 December 2020

Academic Editor: Ozkan Kanat

Copyright (c) 2020 Beata Szymańska et al. This is an open access article distributed under the Creative Commons Attribution License, which permits unrestricted use, distribution, and reproduction in any medium, provided the original work is properly cited.

\begin{abstract}
During the last decade, a significant increase in the incidence of bladder cancer (BC) has been observed. Angiogenesis plays a key role in the process of tumor growth and metastasis. Additionally, the participation of oxidative stress and chronic inflammation in $\mathrm{BC}$ pathogenesis is indicated. The aim of the study was to evaluate the urinary levels of parameters of angiogenesis, stimulating angiogenin (ANG) and inhibiting angiostatin (ANGST), 8-iso-prostaglandin F2 $\alpha$ (8-iso-PGF2 $\alpha$ ) as a marker of oxidative stress, $\gamma$ synuclein (SNCG) as a cancer progression parameter, and interleukin-13 (IL-13) as an anti-inflammatory immunomodulator. The levels of ANG, ANGST, 8-iso-PGF2 $\alpha$, SNCG, and IL-13 in the urine of BC patients and healthy controls were measured by the enzyme-linked immunosorbent assay. These parameters were examined in the whole group of BC patients and in subgroups depending on the clinical stage: nonmuscle-invasive bladder cancer (NMIBC) and muscle-invasive bladder cancer (MIBC); histopathologic malignancy: low grade (LG) and high grade (HG) and in primary and recurrent BC. Significantly, higher urinary parameters were found in BC patients in comparison to controls. Levels of all parameters increased with the development of cancer, with the exception of 8-iso-prostaglandin F2 $\alpha$, in which the level was higher in the early stages of the disease, but these differences were not statistically significant. Some correlations have been demonstrated between parameters in BC patients. Based on the receiver operating characteristic curves, ANG and ANGST had the best diagnostic value for BC. The obtained results indicate the important role of the examined parameters of angiogenesis, oxidative stress, and inflammation in the pathogenesis and development of BC. It is reasonable to continue research in order to thoroughly assess the impact of various associated processes on the course of BC. It is also important to carry out similar tests in patients with other urological diseases.
\end{abstract}

\section{Introduction}

Bladder cancer (BC) is the 10th most common type of cancer worldwide, with an estimated 549,000 new cases and 200,000 deaths in 2018. BC is more common in men than in women, with respective incidence and mortality rates of 9.6 and 3.2 per 100,000 in men (about 4 times higher than for women around the world). Thus, the disease ranks higher among men, in whom it is the sixth most common cancer and ninth leading cause of cancer death. Incidence rates in both sexes are highest in Southern Europe (Greece, with the highest incidence rate in men globally; Spain; Italy), Western Europe (Belgium and the Netherlands), and Northern America [1]. The main reasons that may explain the higher incidence of $\mathrm{BC}$ in more developed countries are faster economic development, intensification of $\mathrm{BC}$ risk factors, including smoking, poor eating habits, and the detrimental effects of environmental pollution, or occupational exposure [2].

Diagnosis of $\mathrm{BC}$ is primarily based on invasive cystoscopy and urinary sediment cytology. Although urinary cytology is used for screening for BC, it is sometimes difficult to judge cytologic specimens, particularly for low-grade 
cancers, while cystoscopy is invasive and burdensome for patients and expensive for healthcare providers [3]. Therefore, new, noninvasive methods for BC detection would open up new possibilities for the diagnosis and monitoring of this disease. Despite the availability of many laboratory biochemical parameters, no marker that would significantly improve the diagnosis of this disease has been found. Therefore, all studies in this area seem to be justified [4].

The development and metastasis of tumors are especially associated with the process of angiogenesis. Tumor angiogenesis occurs when there is an imbalance between factors stimulating and inhibiting this process in favor of proangiogenic factors [5]. Some literature data indicate that tumor angiogenesis, i.e., the formation of new blood vessels within the tumor, plays a significant role in BC pathogenesis [6].

Angiogenin (ANG) is a potent stimulator of new blood vessels through the process of angiogenesis. The study noted the involvement of ANG in the tumor formation process. In addition, there is evidence to support the synthesis of ANG in cancer cells [7]. High levels of this proangiogenic parameter have been found in various cancers: colorectal, breast, kidney, and malignant melanoma. This fact and the demonstration of the relationship between angiogenin and the degree of tumor aggressiveness may indicate a relationship between ANG and the development of cancer, including $\mathrm{BC}$ [8].

Angiostatin (ANGST), the N-terminal fragment of plasminogen, is a powerful antiangiogenic factor produced by the cleavage of plasminogen by several enzymes such as urokinase and tissue plasminogen activator. The role of angiostatin is to inhibit the proliferation and induction of apoptosis of vascular endothelial cells, which can lead to the inhibition of tumor growth. It can also be used in radiotherapy for cancer because of its strong ability to inhibit tumor growth and create new vessels. The function of angiostatin is also to inhibit the activation and migration of neutrophils, which indicates its anti-inflammatory properties [9].

The impact of oxidative stress (OS) on BC development is associated, among other things, with the maintenance of an inflammatory microenvironment that promotes increased proliferation of cancer cells. Studies show that patients with $\mathrm{BC}$ have increased lipid peroxidation, and the products resulting from this process affect not only $\mathrm{BC}$ initiation (through DNA damage as well as lipid and protein oxidation) but also the further stages of cancer progression $[10,11]$. Biomarkers reflecting the process of lipid peroxidation are the most-examined $\mathrm{F} 2$-isoprostane and 8 -iso-prostaglandin F $2 \alpha$ (8-iso-PGF2 $\alpha$ ). Belonging to this group of compounds is considered a reliable parameter for assessing lipid peroxidation in many diseases, such as neurodegenerative diseases, multiple sclerosis, hypercholesterolemia, diabetes, autoimmune diseases, and cancers [12].

Studies show that protein- $\gamma$-synuclein (SNCG) overexpression is an important factor in cancer pathogenesis, stimulating tumor progression through a variety of mechanisms such as promoting cell proliferation and chromosome instability, cell invasion, and metastasis [13]. SNCG is expressed in various types of cancers, and measurement of its levels can be useful as a prognostic parameter in the early identification of the tumor process. This is a factor that can predict unfavorable prognosis in breast, colorectal, and pancreatic cancer, esophageal cancer, prostate, and stomach cancer [14]. Studies on the role of this parameter in bladder cancers have been started $[15,16]$.

Interleukin-13 (IL-13) is a pleiotropic cytokine with antiinflammatory and immunoregulatory activity [17]. Literature data indicate its role in the pathogenesis of cancer, such as breast, ovarian, pancreatic, colorectal, head, and neck cancers and lymphoma cancers $[18,19]$. However, the results of some studies indicate a contradictory role for IL-13 in promoting and fighting the progression of cancer. The involvement of IL-13 in the escape of tumor cells from host immune surveillance is important. The use of IL-13 inhibitors in targeted immunotherapy in cancers is also being considered [20].

The aim of the study was to determine the level of ANG and ANGST as parameters of angiogenesis, 8 -iso-PGF $2 \alpha$ as an indicator of oxidative stress, SNCG as a stimulator of tumor progression, and IL-13 as an inflammatory immunomodulator in the urine of patients with bladder cancer and to estimate their potential as possible diagnostic parameters in these patients.

\section{Materials and Methods}

2.1. Patient Selection and Collection of Data. The study group consisted of BC patients in the Urology and Oncological Urology Department (Wroclaw Medical University) during the period from 2014 to 2015 . The control group was selected from participants with no history of cancer or other chronic inflammation, which was excluded by clinical examination of the cytology of urine sediment and a urine strip test. All participants were informed of the aim of the study and gave written consent to participate. The study was approved by the Ethics Committee of Wroclaw Medical University (KB292/2-16).

Based on histopathological examination of tissues (performed in the Department of Pathomorphology and Oncological Cytology, Wroclaw Medical University), patients were divided into subgroups according to tumor grade: low grade (LG) and high grade (HG) - on the basis of the WHO/International Society of Urological Pathology-ISUP System 2004, into subgroups of NMIBC (nonmuscle-invasive bladder cancer) and MIBC (muscleinvasive bladder cancer), according to the TNM classification (tumor, noduli, and metastases) developed by the Union for International Cancer Control (UICC) in 2009, and additionally according to whether BC was primary or recurrent [4].

2.2. Materials and Methods. The material for the laboratory test examination of selected parameters consisted of urine from $\mathrm{BC}$ patients and the control group. The urine samples were collected in polystyrene containers (Aptaca, Italy) and 
then centrifuged by an MPW-350 laboratory centrifuge (MPW Instruments, Poland) for 10 minutes $(1500 \mathrm{xg})$, after which the obtained supernatant was removed, placed in Eppendorf tubes, and stored at $-80^{\circ} \mathrm{C}$ for further investigation.

Concentrations of selected/chosen parameters were measured in urine by the immunoenzymatic method (ELISA) with enzyme-linked immunosorbent assay kits: Human Angiogenin ELSA Kit, Cusabio, CRL, Human Angiostatin ELISA Kit, Genorise Scientific, USA, 8-Iso-PGF2 $\alpha$-OxiSelect 8 -iso-Prostaglandin F2 $\alpha$ ELISA Kit, Cell Biolabs, USA, Human Gamma-Synuclein (SNCG) ELISA Kit, Cusabio, CRL, and Human IL-13 Platinum ELISA IL-13, eBioscience, Austria, according to the manufacturer's instructions in a listed test.

Concentrations of parameters were calculated in relation to the urine creatinine concentration estimated by Jaffe's routine method based on the reaction of picric acid (Picric Acid, USA, Sigma, Cat. no. 319287).

2.3. Statistical Analysis. Statistical analysis was conducted with Statistica PL software (version 13.3). The normality of distribution was checked by Lilliefors and Kolmogorov-Smirnov tests. The nonparametric $U$ Mann-Whitney and Kruskal-Wallis tests were used for the comparison of the variables between groups, and the post hoc analysis (Bonferroni test) was used for nonparametric data. The associations between continuous variables were analyzed by the Spearman test. The receiver operating characteristic curves (ROCs) were estimated. The area under the curve (AUC) and best cutoff point were calculated. Diagnostic value indicators with $95 \% \mathrm{CI}$ such as sensitivity, specificity, positive predictive value (PPV), negative predictive value $(\mathrm{NPV})$, and likelihood ratio (LR) were calculated. The values of $p<0.05$ were considered as statistically significant.

\section{Results}

3.1. Study Population. The study group consisted of $60 \mathrm{BC}$ patients including 50 men and 10 women with a mean age of 66 years. The control group included 28 healthy volunteers, 23 men and 5 women with a mean age 67 years. No statistically significant differences in characteristic features were observed between patients and the control group. The demographic and clinical characteristics of the examined groups are shown in Table 1. Age and sex of BC patients and the control group were not different $(p>0.05)$.]

\subsection{Comparison of the Level of Urinary Parameters in $B C$} Patients with the Control Group. Median levels and interquartile ranges in $\mathrm{BC}$ patients and the control group, with statistical analysis of examined parameters: ANG, ANGST, 8-iso-PGF2 $\alpha$, SNCG, and IL-13 in the urine, are presented in Table 2 and Figure 1.

As shown in Table 2, median levels of ANG, ANGST, 8iso-PGF2 $\alpha$, SNCG, and IL-13 were 9.3-fold, 6.6-fold, 1.5fold, 1.3-fold, and 1.8-fold higher, respectively, in the BC patients compared with the control group. There were
TABLE 1: Demographic and clinical data of the bladder cancer (BC) patient group and control group.

\begin{tabular}{lcc}
\hline Population characteristic & BC group & Controls \\
\hline$N$ & 60 & 28 \\
Age range (median) & $41-88(66)$ & $50-81(67)$ \\
Male & $50(83 \%)$ & $23(82 \%)$ \\
Female & $10(17 \%)$ & $5(18 \%)$ \\
Clinical grading & & \\
LG & $31(52 \%)$ & \\
HG & $29(48 \%)$ & \\
Clinical subgroups & & \\
NMIBC & $50(83 \%)$ & \\
MIBC & $10(17 \%)$ & \\
Primary cancer & $29(48 \%)$ & \\
Cancer recurrence & $31(52 \%)$ & \\
\hline
\end{tabular}

$N$ : number of patients; LG: low grade; HG: high grade; NMIBC: nonmuscleinvasive bladder cancer; MIBC: muscle-invasive bladder cancer.

statistically significant differences between the levels of all examined parameters (Table 2).

3.3. Assessment of the Diagnostic Value of Urinary Parameters. The ROC curve analysis of ANG, ANGST, 8-iso-PGF2 $\alpha$, SNCG, and IL-13 conducted for examined parameters showed an area under the curve (AUC) of $91 \%$ (95\% confidence interval (CI) $(85-97 \%), p<0.001)$ ), $92 \%$ (95\% CI (87-98\%), $p<0.001)$ ), 67\% (95\% CI (56-78\%), $p=0.003)$ ), $73 \%(95 \%$ CI $(62-84 \%), p<0.001)$ ), and $75 \%$ (95\% CI (65-85\%), $p<0.001)$ ), respectively (Figure 2 ).

Sensitivity, specificity, PPV, NPV, LR+, and LR-for each of the examined parameters are shown in Table 3.

The cutoff value for ANG, ANGST, 8-iso-PGF2 $\alpha$, SNCG, and IL-13 was $0.24 \mathrm{ng} / \mathrm{mg}$ creatinine, $0.33 \mathrm{ng} / \mathrm{mg}$ creatinine, $6.34 \mathrm{ng} / \mathrm{mg}$ creatinine, $13.68 \mathrm{pg} / \mathrm{mg}$ creatinine, and $1.12 \mathrm{pg} /$ $\mathrm{mg}$ creatinine, respectively.

3.4. Relationships between Parameters. In BC patients, significant positive correlations between parameters, ANG vs. $\operatorname{ANGST}(R=0.67, p<0.001)$, SNCG vs. IL-13 $(R=0.60$, $p<0.001), 8$-iso-PGF $2 \alpha$ vs. IL-13 $(R=0.47, p<0.001)$, ANGST vs. 8 -iso-PGF $2 \alpha(R=0.41, p=0.001)$, ANGST vs. IL-13 $(R=0.40, p=0.002)$, and ANGST vs. SNCG $(R=0.36$, $p=0.004)$, were found.

3.5. Dependence of the Parameter Level on the Degree of Malignancy and Invasiveness of BC. Analysis of the levels of the examined parameters was conducted within subgroups of $\mathrm{BC}$ patients divided according to the clinical stage of malignancy. Median levels and interquartile ranges of parameters in the urine of $\mathrm{BC}$ patients in the LG and the $\mathrm{HG}$ subgroups and the control group, with statistical analysis, are presented in Table 4 and Figure 1.

Median levels of ANG, ANGST, 8-iso-PGF2 $\alpha$, SNCG, and IL-13 were 7 -fold, 5 -fold, 1.5 -fold, 1.4 -fold, and 1.6-fold higher, respectively, in the LG subgroup of BC patients compared with the control group. There were statistically significant differences between the levels of all parameters in 
TABLE 2: Median levels and interquartile ranges of examined parameters in the urine of the BC group and control group with statistical analysis.

\begin{tabular}{|c|c|c|c|c|c|}
\hline \multirow{2}{*}{ Parameters } & \multicolumn{2}{|c|}{$\mathrm{BC}$ group } & \multicolumn{2}{|c|}{ Control group } & \multirow{2}{*}{$p$ value } \\
\hline & Median & IQR & Median & IQR & \\
\hline ANG (ng/mg cr.) & 0.58 & $0.31-1.04$ & 0.06 & $0.04-0.15$ & $<0.001 *$ \\
\hline ANGST (ng/mg cr.) & 0.53 & $0.28-0.91$ & 0.08 & $0.06-0.16$ & $<0.001 *$ \\
\hline 8 -Iso-PGF $2 \alpha$ (ng/mg cr.) & 5.11 & $2.90-25.58$ & 3.44 & $2.74-4.58$ & $0.013 *$ \\
\hline SNCG (pg/mg cr.) & 15.84 & $13.05-26.88$ & 12.41 & $9.06-16.07$ & $0.001 *$ \\
\hline IL-13 (pg/mg cr.) & 1.81 & $1.23-3.21$ & 1.01 & $0.68-1.58$ & $<0.001 *$ \\
\hline
\end{tabular}

ANG: angiogenin; ANGST: angiostatin; 8-Iso-PGF2 $\alpha$ : 8-iso-prostaglandin F2 $\alpha$; SNCG: $\gamma$-synuclein; IL-13: interleukin-13; IQR: interquartile ranges. $*$ Statistically significant difference between BC and the control group ( $U$ Mann-Whitney test).

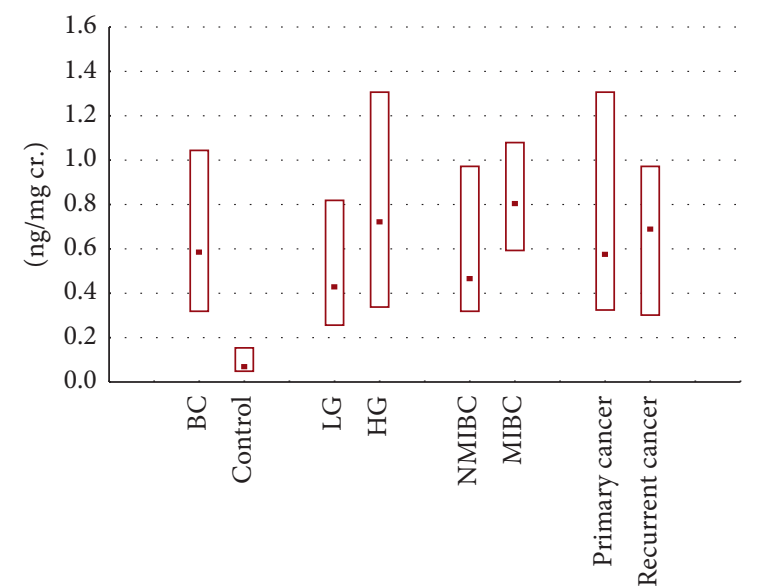

- Mediana

$\square 25 \%-75 \%$

(a)

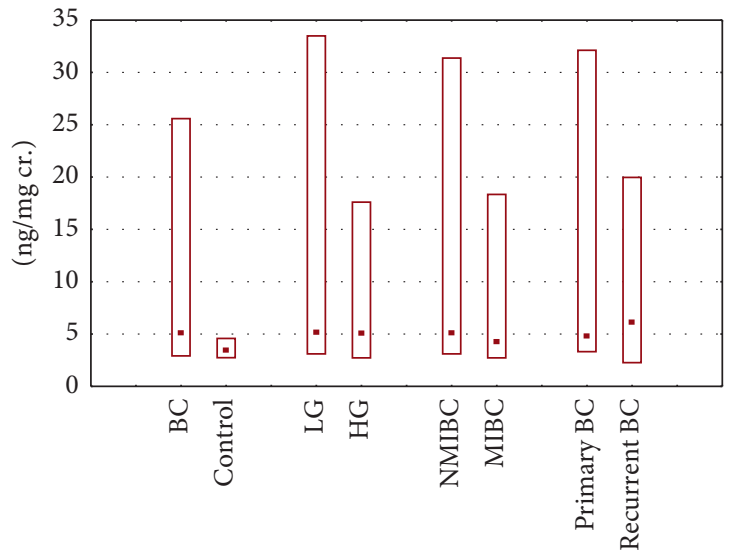

Mediana

$\square 25 \%-75 \%$

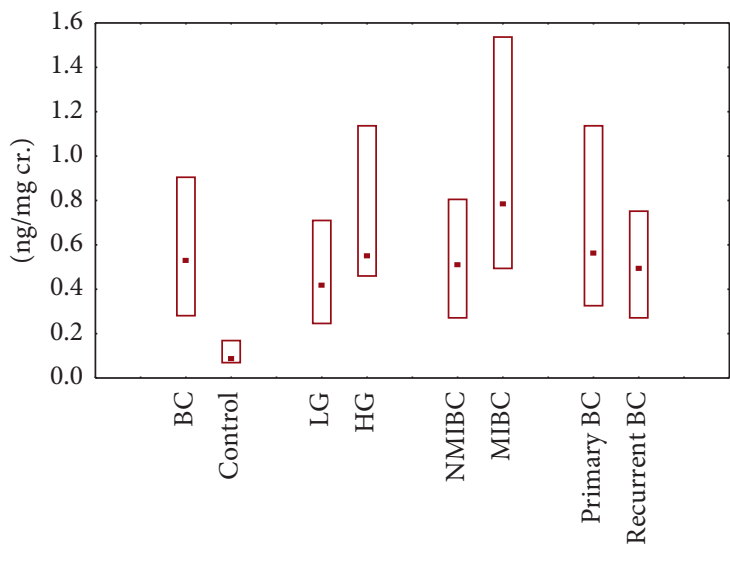

- Mediana

$25 \%-75 \%$

(b)

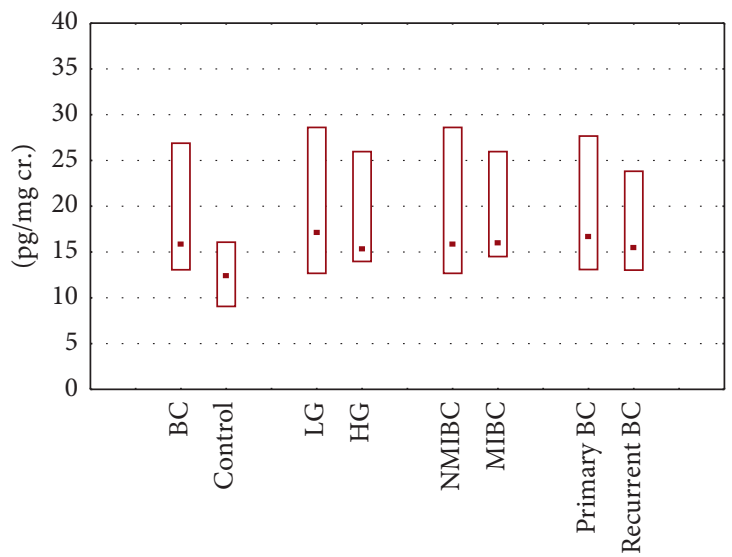

- Mediana

$25 \%-75 \%$

(c)

Figure 1: Continued. 


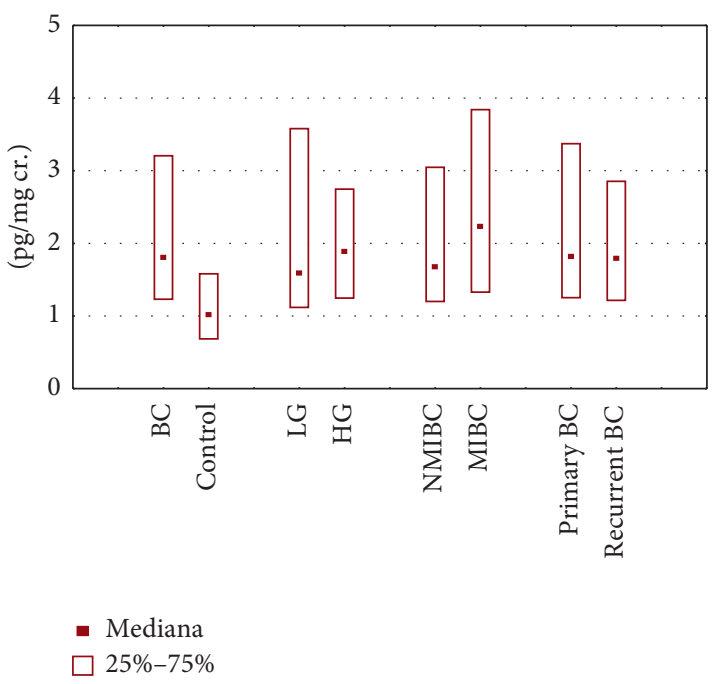

(e)

Figure 1: Median levels and interquartile ranges of examined parameters in the urine in all patient groups and the control group: (a) angiogenin, (b) angiostatin, (c) 8-iso-prostaglandin F2 $\alpha$, (d) $\gamma$-synuclein, and (e) interleukin-13.

the LG subgroup of $\mathrm{BC}$ patients and those of the control group. Median levels of ANG, ANGST, 8-iso-PGF2 $\alpha$, SNCG, and IL-13 were 12 -fold, 6.6 -fold, 1.5 -fold, 1.2 -fold, and 1.9fold higher, respectively, in the HG subgroup of BC patients compared with the control group. There were statistically significant differences between the levels of parameters in the HG subgroup of $\mathrm{BC}$ patients and in the control group without 8 -iso-PGF $2 \alpha$. However, statistical analysis showed no significant differences between any parameter levels in the LG or the HG subgroups of BC patients (Table 4).

Median levels and interquartile ranges of parameters in the urine of $\mathrm{BC}$ patients in the NMIBC and the MIBC subgroups and the control group, with statistical analysis, are shown in Table 5 and Figure 1.

Median levels of ANG, ANGST, 8-iso-PGF2 $\alpha$, SNCG, and IL-13 were 7.7-fold, 6.3-fold, 1.5-fold, 1.3-fold, and 1.7fold higher, respectively, in the NMIBC subgroup of BC patients compared with the control group. There were statistically significant differences between the levels of all parameters in the NMIBC subgroup of BC patients and those in the control group. Median levels of ANG, ANGST, 8-iso-PGF2 $\alpha$, SNCG, and IL-13 were 13.3-fold, 9.8-fold, 1.2fold, 1.3-fold, and 2.2-fold higher, respectively, in the MIBC subgroup of $\mathrm{BC}$ patients compared with the control group. There were statistically significant differences between the levels of examined parameters in the MIBC subgroup of BC patients and the control group, except for 8-iso-PGF2 $\alpha$. The analysis showed no significant differences between any parameter levels in the NMIBC or the MIBC subgroups of BC patients (Table 5).

3.6. Differences in the Level of Parameters in Primary and Recurrent $B C$. Median levels and interquartile ranges of parameters in the urine of $\mathrm{BC}$ patients in the subgroup of primary $\mathrm{BC}$, the subgroup of recurrent $\mathrm{BC}$, and the control group, with statistical analysis, are presented in Table 6 and Figure 1.

Median levels of ANG, ANGST, 8-iso-PGF2 $\alpha$, SNCG, and IL-13 were 9.5-fold, 7-fold, 1.4-fold, 1.3-fold, and 1.8fold higher, respectively, in the subgroup of primary BC patients compared with the control group. There were statistically significant differences between the levels of parameters in the subgroup of primary $\mathrm{BC}$ patients and the control group without 8-iso-PGF2 $\alpha$. Median levels of ANG, ANGST, 8-iso-PGF2 $\alpha$, SNCG, and IL-13 were 11.3-fold, 6.1fold, 1.7-fold, 1.2-fold, and 1.8-fold higher, respectively, in the subgroup of recurrent $\mathrm{BC}$ patients compared with the control group. There were statistically significant differences between the levels of parameters in the subgroup of primary $\mathrm{BC}$ patients and those of the control group, except for 8-isoPGF2 $\alpha$. Additionally, the analysis showed no significant differences between any parameter levels in the NMIBC and the MIBC subgroups of $B C$ patients.

\section{Discussion}

Angiogenesis is an essential part of many physiological processes, but it is also critical to tumor growth and tumor cell survival as it ensures a constant supply of oxygen and nutrients. In the case of cancer, this condition is disturbed, and there is a shift towards proangiogenic factors [6].

In our research, the results were quite surprising because the levels of ANG and ANGST in the urine of BC patients were similar, which may indicate a balance between these pro- and antiangiogenic parameters. However, it should be noted that these parameters were determined in the urine of patients and reflected the local urothelial $\mathrm{BC}$ angiogenesis process. An increase in the levels of ANG and ANGST was observed with an increase in the invasiveness and malignancy of cancer (MIBC and HG), but it was not statistically significant. In primary cancer and recurrence, ANG and 


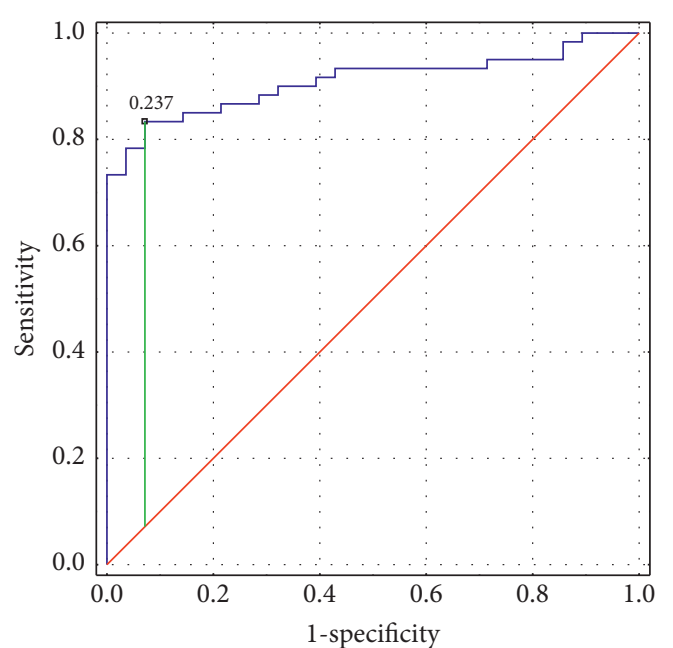

(a)

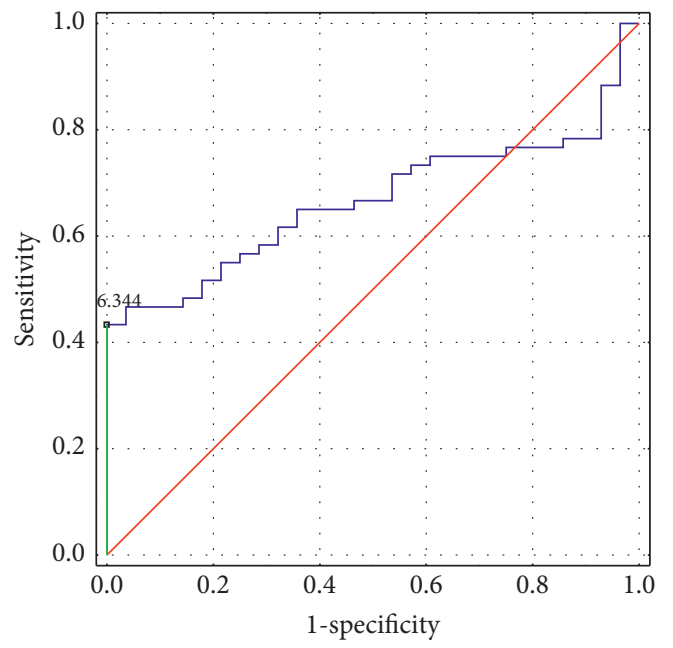

(c)

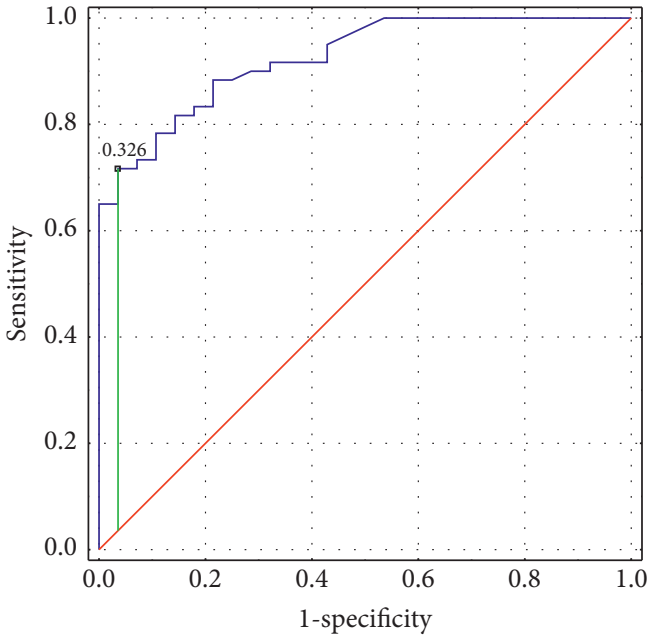

(b)

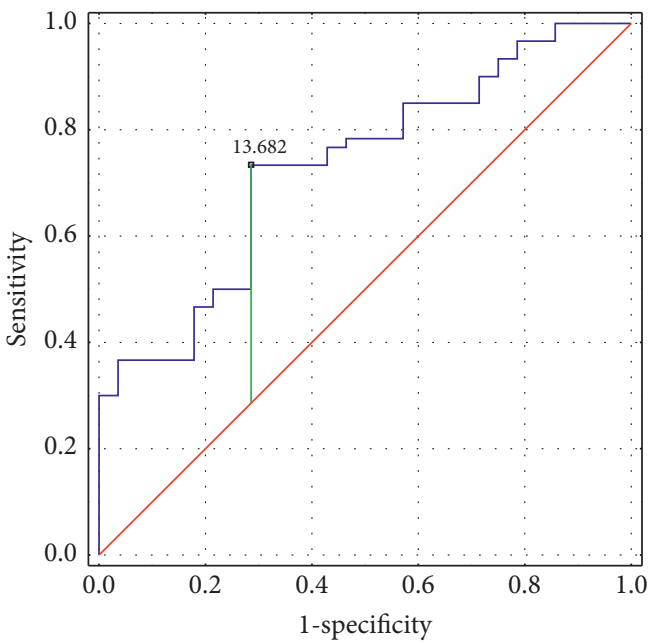

(d)

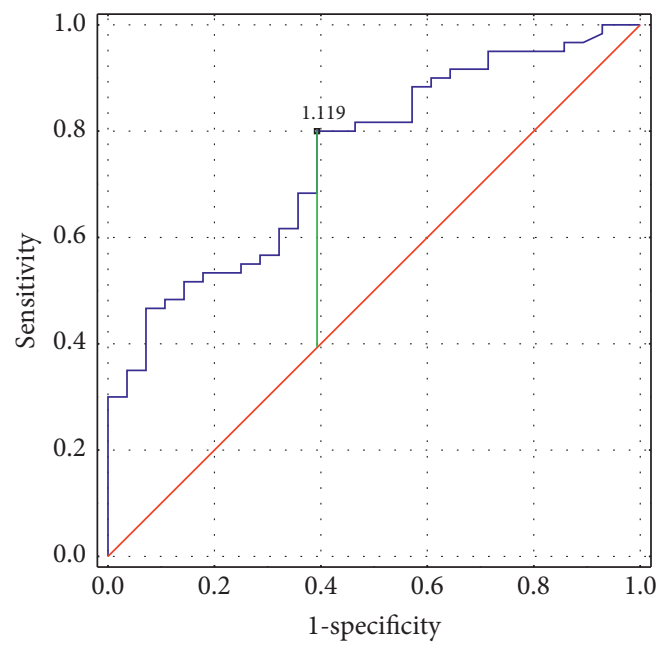

(e)

FIGURE 2: ROC curve analysis for examined parameters: (a) angiogenin, (b) angiostatin, (c) 8-iso-prostaglandin F2 $\alpha$, (d) $\gamma$-synuclein, and (e) interleukin-13. 
TABLE 3: Diagnostic value of examined parameters.

\begin{tabular}{lcccccc}
\hline $\begin{array}{l}\text { Diagnostic value of } \\
\text { parameters }\end{array}$ & Se (\%) (CI 95\%) & Sp (\%) (CI 95\%) & PPV (\%) (CI 95\%) & NPV (\%) (CI 95\%) & LR+ (CI 95\%) & LR- (CI 95\%) \\
\hline ANG (ng/mg cr.) & $83.3(71.5-91.0)$ & $92.9(76.5-99.1)$ & $96.2(86.7-99.0)$ & $72.2(59.4-82.2)$ & $11.7(3.1-44.6)$ & $0.2(0.1-0.3)$ \\
ANGST (ng/mg cr.) & $71.7(58.6-82.5)$ & $96.4(81.7-99.9)$ & $97.7(86.2-99.7)$ & $61.4(51-70.5)$ & $(2.9-138.4)$ & $0.2(0.2-0.4)$ \\
& & & & & & \\
8-Iso-PGF2 $\alpha$ (ng/mg cr.) & $45.0(32.1-58.4)$ & $96.4(81.7-99.9)$ & $96.4(79.4-99.5)$ & $45.0(39.2-51.0)$ & $12.6(1.8-88.1)$ & $0.6(0.4-0.7)$ \\
SNCG (pg/mg cr.) & $71.7(58-82.5)$ & $71.4(51.3-86.8)$ & $84.3(74.6-90.8)$ & $54.1(42.5-65.2)$ & $2.5(1.4-4.6)$ & $0.4(0.2-0.6)$ \\
IL-13 (pg/mg cr.) & $80.1(67.7-89.2)$ & $60.7(40.6-78.5)$ & $81.4(73.0-87.6)$ & $58.6(44.1-71.8)$ & $2.0(1.3-3.3)$ & $0.3(0.2-0.6)$ \\
\hline
\end{tabular}

ANG: angiogenin; ANGST: angiostatin; 8-Iso-PGF2 $\alpha$ : 8-iso-prostaglandin F2 $\alpha$; SNCG: $\gamma$-synuclein; IL-13: interleukin-13; Se: sensitivity; Sp: specificity; PPV: positive predictive value; NPV: negative predictive value; LR+: positive likelihood ratio; LR-: negative likelihood ratio; CI: confidence interval.

TABLE 4: Median levels and interquartile ranges of parameters in the urine of the LG (A) subgroup, HG (B) subgroup, and control group (C) with statistical analysis.

\begin{tabular}{|c|c|c|c|c|c|c|c|c|}
\hline \multirow{3}{*}{ Parameters } & \multicolumn{8}{|c|}{ Subgroups } \\
\hline & \multicolumn{2}{|c|}{ LG (A) } & \multicolumn{2}{|c|}{ HG (B) } & \multicolumn{2}{|c|}{ Control group (C) } & \multirow{2}{*}{$p$ value } & \multirow{2}{*}{ Post hoc analysis } \\
\hline & $\mathrm{Me}$ & IQR & $\mathrm{Me}$ & IQR & $\mathrm{Me}$ & IQR & & \\
\hline ANG (ng/mg cr.) & 0.42 & $0.25-0.81$ & 0.72 & $0.33-1.31$ & 0.06 & $0.04-0.15$ & $<0.001$ & $\begin{array}{c}\mathrm{A}: \mathrm{C}<0.001 * \\
\mathrm{~B}: \mathrm{C}<0.001 * \\
\mathrm{~A}: \mathrm{B}=\mathrm{NS}\end{array}$ \\
\hline ANGST (ng/mg cr.) & 0.41 & $0.24-0.71$ & 0.54 & $0.45-1.13$ & 0.08 & $0.06-0.16$ & $<0.001$ & $\begin{array}{c}\mathrm{A}: \mathrm{C}<0.001 * \\
\mathrm{~B}: \mathrm{C}<0.001 * \\
\mathrm{~A}: \mathrm{B}=\mathrm{NS}\end{array}$ \\
\hline 8-Iso-PGF2 $\alpha$ (ng/mg cr.) & 5.15 & $3.10-33.48$ & 5.07 & $2.72-17.60$ & 3.44 & $2.74-4.58$ & 0.03 & $\begin{array}{c}A: C=0.03 * \\
B: C=N S \\
A: B=N S\end{array}$ \\
\hline SNCG (pg/mg cr.) & 17.11 & $12.67-28.61$ & 15.32 & $13.97-25.97$ & 12.41 & $9.06-16.07$ & 0.002 & $\begin{array}{c}A: C=0.004 * \\
B: C=0.013 * \\
A: B=N S\end{array}$ \\
\hline IL-13 (pg/mg cr.) & 1.58 & $1.11-3.57$ & 1.88 & $1.24-2.75$ & 1.01 & $0.68-1.58$ & $<0.001$ & $\begin{array}{c}A: B=0.004 * \\
B: C=0.002 * \\
A: B=N S\end{array}$ \\
\hline
\end{tabular}

ANG: angiogenin; ANGST: angiostatin; 8-Iso-PGF2 $\alpha$ : 8-iso-prostaglandin F2 $\alpha$; SNCG: $\gamma$-synuclein; IL-13: interleukin-13; LG: low grade; HG: high grade; Me: median; CI: confidence interval; NS: not statistically significant. $*$ Statistically significant difference between LG (A), HG (B), and control group (C) using Kruskal-Wallis test.

TABLE 5: Median levels and interquartile ranges of examined parameters in the urine of the NMIBC (A) subgroup, MIBC (B) subgroup, and control group with statistical analysis.

\begin{tabular}{|c|c|c|c|c|c|c|c|c|}
\hline \multirow{3}{*}{ Parameters } & \multicolumn{8}{|c|}{ Subgroups } \\
\hline & \multicolumn{2}{|c|}{ NMIBC (A) } & \multicolumn{2}{|c|}{ MIBC (B) } & \multicolumn{2}{|c|}{ Control group (C) } & \multirow{2}{*}{$p$ value } & \multirow{2}{*}{ Post hoc analysis } \\
\hline & $\mathrm{Me}$ & IQR & $\mathrm{Me}$ & IQR & $\mathrm{Me}$ & IQR & & \\
\hline ANG (ng/mg cr.) & 0.46 & $0.31-0.97$ & 0.80 & $0.59-1.07$ & 0.06 & $0.04-0.15$ & $<0.001$ & $\begin{array}{c}\mathrm{A}: \mathrm{C}<0.001 * \\
\mathrm{~B}: \mathrm{C}<0.001 * \\
\mathrm{~A}: \mathrm{B}=\mathrm{NS}\end{array}$ \\
\hline ANGST (ng/mg cr.) & 0.50 & $0.27-0.80$ & 0.78 & $0.49-1.53$ & 0.08 & $0.06-0.16$ & $<0.001$ & $\begin{array}{c}\mathrm{A}: \mathrm{C}<0.001 * \\
\mathrm{~B}: \mathrm{C}<0.001 * \\
\mathrm{~A}: \mathrm{B}=\mathrm{NS}\end{array}$ \\
\hline 8-Iso-PGF $2 \alpha$ (ng/mg cr.) & 5.11 & $3.10-31.36$ & 4.27 & $2.72-18.34$ & 3.44 & $2.74-4.58$ & 0.04 & $\begin{array}{c}A: C=0.03 * \\
B: C=N S \\
A: B=N S\end{array}$ \\
\hline SNCG (pg/mg cr.) & 15.83 & $12.67-28.61$ & 16.00 & $14.50-25.97$ & 12.41 & $9.06-16.07$ & 0.002 & $\begin{array}{c}A: C=0.004 * \\
B: C=0.024 * \\
A: B=N S\end{array}$ \\
\hline IL-13 (pg/mg cr.) & 1.67 & $1.20-3.04$ & 2.23 & $1.32-3.84$ & 1.01 & $0.68-1.58$ & $<0.001$ & $\begin{array}{c}\mathrm{A}: \mathrm{B}=0.002 * \\
\mathrm{~B}: \mathrm{C}=0.004 * \\
\mathrm{~A}: \mathrm{B}=\mathrm{NS}\end{array}$ \\
\hline
\end{tabular}

ANG: angiogenin; ANGST: angiostatin; 8-Iso-PGF2 $\alpha$ : 8-iso-prostaglandin F2 $\alpha$; SNCG: $\gamma$-synuclein; IL-13: interleukin-13; NMIBC: nonmuscle-invasive bladder cancer; MIBC: muscle-invasive bladder cancer; Me: median; CI: confidence interval; NS: not statistically significant. $*$ Statistically significant difference between NMIBC (A), MIBC (B), and control group (C) using Kruskal-Wallis test. 
TABLE 6: Median levels and interquartile ranges of examined parameters in the urine of the BC primary cancer (A) subgroup, the cancer recurrent (B) subgroup, and the control group (C) with statistical analysis.

\begin{tabular}{|c|c|c|c|c|c|c|c|c|}
\hline \multirow{3}{*}{ Parameters } & \multicolumn{8}{|c|}{ Subgroups } \\
\hline & \multicolumn{2}{|c|}{ Primary cancer (A) } & \multicolumn{2}{|c|}{ Cancer recurrent (B) } & \multicolumn{2}{|c|}{ Control group (C) } & \multirow{2}{*}{$p$ value } & \multirow{2}{*}{ Post hoc analysis } \\
\hline & $\mathrm{Me}$ & IQR & $\mathrm{Me}$ & IQR & $\mathrm{Me}$ & IQR & & \\
\hline ANG (ng/mg cr.) & 0.57 & $0.32-1.31$ & 0.68 & $0.30-0.92$ & 0.06 & $0.04-0.15$ & $<0.001$ & $\begin{array}{c}\mathrm{A}: \mathrm{C}<0.001 * \\
\mathrm{~B}: \mathrm{C}<0.001 * \\
\mathrm{~A}: \mathrm{B}=\mathrm{NS}\end{array}$ \\
\hline ANGST (ng/mg cr.) & 0.56 & $0.33-1.14$ & 0.49 & $0.27-0.75$ & 0.08 & $0.06-0.16$ & $<0.001$ & $\begin{array}{c}\mathrm{A}: \mathrm{C}<0.001 * \\
\mathrm{~B}: \mathrm{C}<0.001 * \\
\mathrm{~A}: \mathrm{B}=\mathrm{NS}\end{array}$ \\
\hline 8-Iso-PGF $2 \alpha$ (ng/mg cr.) & 4.79 & $3.31-32.11$ & 5.89 & $2.26-19.97$ & 3.44 & $2.74-4.58$ & 0.04 & $\begin{array}{l}A: B=N S \\
B: C=N S \\
A: B=N S\end{array}$ \\
\hline SNCG (pg/mg cr.) & 16.68 & $13.09-27.67$ & 15.46 & $13.02-23.31$ & 12.41 & $9.06-16.07$ & 0.002 & $\begin{array}{c}A: B=0.006 * \\
B: C=0.009 * \\
A: B=N S\end{array}$ \\
\hline IL-13 (pg/mg cr.) & 1.81 & $1.25-3.37$ & 1.79 & $1.21-2.85$ & 1.01 & $0.68-1.58$ & $<0.001$ & $\begin{array}{c}A: B=0.004 * \\
B: C=0.002 * \\
A: B=N S\end{array}$ \\
\hline
\end{tabular}

ANG: angiogenin; ANGST: angiostatin; 8-Iso-PGF2 $\alpha$ : 8-iso-prostaglandin F2 $\alpha$; SNCG: $\gamma$-synuclein; IL-13: interleukin-13; NMIBC: nonmuscle-invasive bladder cancer; MIBC: muscle-invasive bladder cancer; Me: median; CI: confidence interval; NS: not statistically significant. $*$ Statistically significant difference between primary cancer (A), cancer recurrence (B), and control group (C) using Kruskal-Wallis test.

ANGST levels were similar. ROC analysis also indicated ANG and ANGST to be the parameters with the best diagnostic value in $\mathrm{BC}$ among those examined. The studies showed a strong mutual positive correlation between ANG and ANGST $(R=0.67, p<0.001)$. This confirms the significant role of angiogenesis processes in the development and metastasis of tumors [6].

Peres et al. [21], in studies on human BC cell lines (UROtsa, RT4, RT112, 5637, UM-UC-3, T24, TCCSUP, and UM-UC-14), proved that high ANG expression correlated with the increased matrix activity of metalloproteinase-2 (MMP2). In patients with MIBC, they showed higher levels of ANG and MMP2 and lower levels of these parameters compared to NMIBC. The average relative levels of mRNA for both ANG and MMP2 in invasive BC were significantly increased compared to the average relative levels of ANG and MMP2 mRNA in noninvasive BC.

Urquidi et al. [22] examined the concentration of ANG in the urine of a group of patients with active $\mathrm{BC}$ and people with benign urological disorders. They observed a significantly higher level of ANG in BC compared to benign urological diseases. Angiogenesis plays a role in both local tumor development and distant metastases. ANG appears to be a key stimulant in the process of angiogenesis, enabling tumor growth as well as metastasis.

No information was found in the available literature regarding the research into ANGST in BC, so our observations are innovative.

In our research, the effect of oxidative stress on the cancer process in the bladder was observed, and the elevated level of 8 -iso-PGF $2 \alpha$ in the urine of BC patients suggests an increase in lipid peroxidation, especially in the early stages of the disease. Based on the analysis of our own results carried out in subgroups of BC patients with different degrees of clinical advancement and histopathological malignancy, higher average 8 -iso-PGF2 $\alpha$ values were found in benign BC (NMIBC and LG) conditions compared to advanced ones (MIBC and $\mathrm{HG}$ ), but they were not statistically significant. Additionally, the 8-Iso-PGF2 $\alpha$ values in recurrent BC were slightly higher than in primary $\mathrm{BC}$, although not statistically significant.

Verratti et al. [23], using the immunocytochemical method, examined the expression of 8 -iso-PGF $2 \alpha$ in the healthy urinary bladder tissue and the tissue derived from BC. Release of 8 -iso-PGF $2 \alpha$ was significantly reduced in the tumor tissue compared to the healthy bladder tissue. Inhibiting the production of a strong vasoconstrictor such as 8 -iso-PGF2 $\alpha$ in the vascular homeostatic mechanism of the bladder may reflect tumor response, tending to contrast the vasoconstrictor effect and the need to support oxygen supply to cancer cells.

SNCG is secreted from different tumor cells. It is a parameter that has recently aroused interest among researchers. Only single literature study indicates the usefulness of this marker in the diagnosis of bladder cancer [16].

Mhawech-Fauceglia et al. [24], using enzyme immunoassay and western blotting, were the first to discover high SNCG expression in NMIBC and confirm its high diagnostic performance by high AUC value. The researchers also showed, for the first time, a relationship between high SNGC expression and the tumor stage in BC patients. Additionally, the authors indicated that urine SNCG can discriminate BC from other urinary diseases and is a useful prognosticator of postsurgical recurrence.

Liu et al. [16] revealed that the level of SNCG in urine among patients who had BC tumor resection was significantly reduced. In addition, in patients who were to experience a relapse in the future, the marker concentration was higher than among those who did not relapse. 
Zhao and Xing [15] studied the SNCG expression, using immunohistochemistry, in tissues from BC patients and revealed statistically significantly higher expression of this parameter in samples taken from patients than in the tissues of healthy individuals, especially at higher stages of cancer.

In our study, SNCG levels were statistically significantly higher in BC and in all analyzed subgroups compared to controls; however, no significant differences were found between the initial and more advanced stages of BC. The studies showed a strong mutual positive correlation between SNCG and IL-13 $(R=0.60, p<0.001)$.

Our research has shown higher levels of IL-13 in more advanced stages of the disease (HG and MIBC). The increased presence of IL-13 in the urine of BC patients was probably due to the increased inflammatory process associated with cancer, which includes local changes in the urinary bladder or may have its origin in the tumor itself. Tumor cells produce IL-13 in response to the host's defense response and defend against apoptosis [25].

Margel et al. [26] showed an elevated concentration of IL-13 in the urine of BC patients compared to the control, but no statistically significant differences between MIBC and NMIBC were revealed, although higher IL-13 values were obtained for MIBC, similarly to our studies.

The study conducted by Mousa et al. [27] aimed at detecting IL-13 expression in BC tumor tissues and explaining the relationship between its expression and the clinical form of cancer. Expression of IL-13 in tumor cells has been studied in patients with bladder cancer at various stages of clinical malignancy and patients with benign bladder tumors. Tissue sections were analyzed by immunohistochemistry for the presence of IL-13. The results showed a high positive expression of IL-13 in high-grade cancer tissues compared to tissues from benign tumors, which confirmed that IL-13 is involved in tumor progression. High levels of IL-13 provoke reduced tumor immune control, which leads to unrestrained tumor growth. Involved in immune surveillance, the IL-13 tumor contributes to escape from apoptosis and strengthens its growth [28].

To summarize, the obtained results indicate the important role of the examined parameters of angiogenesis, oxidative stress, and inflammation in the pathogenesis and development of BC. Additionally, differences in terms of structure and function revealed by the mutual correlations between examined parameters in the urine in BC patients may indicate their complicity in the tumor process occurring within urinary bladder cancer.

A major aim in the management of urothelial carcinoma is the prevention of the recurrence and progression of the disease. For patients with an intermediate-to-high risk of recurrence, the standard treatment consists of intravesical instillations with bacillus Calmette-Guérin (BCG) to prevent or delay tumor recurrence and progression [29].

Ferro et al. [30] studied the association of baseline counts of basophils, eosinophils, and monocytes with outcomes of patients with high-grade $\mathrm{T} 1$ bladder cancer receiving a standard course of intravesical BCG. Baseline basophil count may predict recurrence in BCG-treated HG/G3 T1 bladder cancer patients. Basophils may have a role in BCG-treated bladder cancer patients because of their ability to facilitate Th2 polarization by secreting proinflammatory interleukins (IL-4 or IL-13) and skewing antigen-presenting cells towards a type 2 response.

Angiogenesis is a crucial step for tumor growth and progression in almost all types of cancers, including BC [31]. In cancer tissues, microvessel density (MVD) is commonly used to evaluate the angiogenic status. MVD was found to be closely associated with recurrence after intravesical BCG therapy [32].

BCG serves as a free radical generator. BCG generates $\mathrm{H}_{2} \mathrm{O}_{2}$ which serves as the trigger for COS and cell damage. Production of $\mathrm{H}_{2} \mathrm{O}_{2}$ by $\mathrm{BCG}$, following binding to and internalization by $\mathrm{BC}$ cells, sets the stage for a second wave of cell-generated oxidants involving iNOS [33]. Free radicals (superoxide and $\mathrm{NO}$ ) and reactive molecules potentiate intracellular signaling pathways and downstream gene expression and result in a cellular phenotype that defines the BCG treatment effect. Loss of BCG viability is associated with decreased $\mathrm{H}_{2} \mathrm{O}_{2}$ production, inefficient induction of $\mathrm{COS}$ and its direct consequences, and decreased treatment efficacy [34]. NO is considered one of the main factors responsible for the cytotoxic activity that macrophages exert against tumor cells [31].

Based on the data presented above, the use of the urinary parameter panel (characterizing processes such as angiogenesis, immune response, and oxidative stress) seems to be useful in assessing the effectiveness of BCG therapy in patients with BC. The increase in IL-13, ANG, and SNCG in the urine of $\mathrm{BC}$ patients may be an unfavorable prognostic factor in BCG therapy, in contrast to ANGST and 8-iso-PGF2 $\alpha$, proving the treatment effectiveness.

Obesity and metabolic disorder such as type 2 diabetes mellitus (T2DM) have been identified as a major risk factor associated with cancers, including BC. The incidence of cancer due to obesity is estimated to be approximately $20 \%$ of all causes of cancers [35].

Ferro et al. [36] investigated the prognostic role of T2DM in patients with primary T1HG/G3 nonmuscle-invasive bladder cancer (NMIBC) treated with transurethral resection of the bladder (TURB) and BCG therapy. The authors showed that prior history of T2DM was significantly associated with a greater risk of disease recurrence and disease progression to muscle-invasive $\mathrm{BC}$, as well as with overall and cancer-specific survival.

Meta-analysis of 14 prospective cohort studies showed a nonlinear positive relationship between the BMI and bladder cancer suggesting that each $5 \mathrm{~kg} / \mathrm{m}^{2}$ increase of the BMI corresponded to a $3.1 \%$ increase of the bladder cancer risk, especially when the BMI exceeded $30 \mathrm{~kg} / \mathrm{m}^{2}$ [37].

Ferro et al. [38] evaluated the impact of the BMI on survival in patients with high-risk nonmuscle-invasive bladder cancer. In particular, they showed that overweight and obesity were associated with a greater risk of progression. Instead, a higher risk of recurrence was demonstrated only for obese patients. The association of obesity with a poor clinical outcome in T1G3 NMIBC could be explained on the basis of several factors. In particular, it is well known that obesity is characterized by insulin resistance and low- 
grade systemic inflammation, which may affect the oncological outcomes of NMIBC patients as a result of insulin, insulin-like growth factor 1(IGF-1), cytokines, and growth factor effects. Furthermore, obesity is associated with high levels of inflammatory cytokines, such as leptin, IL-6, and TNF- $\alpha$, produced by adipocytes and immune cells infiltrating adipose tissue.

To date, there is no reliable method for the prediction of response to chemotherapy, resulting in a possible overtreatment in nonresponders with unnecessary toxicity that might render patients in a deteriorated physical condition without the opportunity for additional, alternative therapy [39]. The parameters presented in our study, determined in the urine of patients with $\mathrm{BC}$, reflect the multifaceted processes occurring during the course of the disease, such as angiogenesis, oxidative stress, and immune response. So far, a similar parameter panel has not been investigated in a possible evaluation of the effectiveness of chemotherapy and immunotherapy in bladder cancer. Considering the noninvasive nature of the tests (urine tests), which are an important alternative to invasive tests, it seems interesting to investigate the changes in the concentration of selected parameters before and during the subsequent stages of the therapy. Such studies could assess the multidirectional influence of chemotherapy and immunotherapy in BC.

\section{Conclusion}

The levels of selected urinary parameters (ANG, ANGST, 8iso-PGF $2 \alpha$, SNCG, and IL-13) were statistically significantly higher than the levels of these parameters observed in the control group. The presented results may suggest the association of oxidative stress (reflected by lipid peroxidation), angiogenesis process, or inflammation in bladder cancer pathogenesis and the development of this disease. It is reasonable to continue research in order to thoroughly assess the impact of various associated processes on the course of this disease.

The possibility of using a panel of selected urinary BC parameters to assess the effectiveness of BCG therapy should also be considered.

\section{Data Availability}

The datasets generated and/or analyzed during the current study are available from the corresponding author upon reasonable request.

\section{Ethical Approval}

This study was performed in line with the principles of the Declaration of Helsinki. Approval was granted by the Ethics Committee of Wroclaw Medical University (KB-292/2-16).

\section{Consent}

Informed consent was obtained from all individual participants included in the study.

\section{Conflicts of Interest}

The authors declare that they have no conflicts of interest.

\section{Authors' Contributions}

All authors contributed to the study conception and design. Material preparation, data collection, and analysis were performed by Beata Szymańska, Ewa Sawicka, and Michal Matuszewski. The first draft of the manuscript was written by Beata Szymańska. Revising it critically for important intellectual content and final approval of the version to be submitted were done by Agnieszka Piwowar and Janusz Dembowski. All authors read and approved the final manuscript.

\section{References}

[1] F. Bray, J. Ferlay, I. Soerjomataram, R. Siegel, L. A. Torre, and A. Jemal, "Global cancer statistics 2018: GLOBOCAN estimates of incidence and mortality worldwide for 36 cancers in 185 countries," A Cancer Journal for Clinicians, vol. 68, no. 2, pp. 394-424, 2018.

[2] W. W. L. Cheung, C. Leung, M. C. S. Wong, F. D. H. Fung, C. F. Ng, and W. B. Goggins, "The global epidemiology of bladder cancer: a joinpoint regression analysis of its incidence and mortality trends and projection," Scientific Reports, vol. 8, no. 1, Article ID 129, 2018.

[3] J. Leal, R. Luengo-Fernandez, R. Sullivan, and J. A. Witjes, "Economic burden of bladder cancer across the European Union," European Urology, vol. 69, no. 3, pp. 438-447, 2006, https://www.researchgate.net/deref/http\%3A\%2F\%2Fdx.doi. org\%2F10.1016\%2Fj.eururo.2015.10.024.

[4] J. J. D’Costa, D. G. Ward, and R. T. Bryan RT, "Urinary biomarkers for the diagnosis of urothelial bladder cancer," New Horizons in Translational Medicine, vol. 3, no. 2, pp. 221-223, 2017.

[5] B. Döme, M. J. C. Hendrix, S. Paku, J. Tóvári, and J. Tímár, "Alternative vascularization mechanisms in cancer," The American Journal of Pathology, vol. 170, no. 1, pp. 1-15, 2007.

[6] L. P. Fus and B. Górnicka, "Role of angiogenesis in urothelial bladder carcinoma," Central European Journal of Urology, vol. 69, no. 4, pp. 258-263, 2016.

[7] A. Tello-Montoliu, J. V. Patel, and G. Y. H. Lip, “Angiogenin: a review of the pathophysiology and potential clinical applications," Journal of Thrombosis and Haemostasis, vol. 4, no. 1, pp. 1864-1874, 2006.

[8] S. Fang, H. Repo, H. Joensuu, A. Orpana, and P. Salven, "High serum angiogenin at diagnosis predicts for failure on longterm treatment response and for poor overall survival in nonHodgkin lymphoma," European Journal of Cancer, vol. 47, no. 2, pp. 1708-1716, 2011.

[9] T. Li, G. Kang, T. Wang, and H. Huang, "Tumor angiogenesis and anti-angiogenic gene therapy for cancer," Oncology Letters, vol. 16, no. 1, pp. 687-702, 2018.

[10] E. Sawicka, A. Lisowska, P. Kowal, and A. Długosz, "The role of oxidative stress in bladder cancer," Postępy Higieny $i$ Medycyny Doświadczalnej, vol. 69, no. 2, pp. 744-752, 2015.

[11] G. L. Milne, Q. Dai, and L. J. Roberts, “The isoprostanes-25 years later," Biochimica et Biophysica Acta-Molecular and Cell Biology of Lipids, vol. 1851, no. 5, pp. 433-445, 2014.

[12] G. L. Milne, S. C. Sanchez, E. S. Musiek, and J. D. Morrow, "Quantification of F2-isoprostanes as a biomarker of oxidative stress," Nature Protocols, vol. 2, no. 1, pp. 221-226, 2007. 
[13] A. Strohl, K. Mori, S. Akers et al., "Synuclein- $\gamma$ (SNCG) expression in ovarian cancer is associated with high-risk clinicopathologic disease," Journal of Ovarian Research, vol. 9, no. 2, pp. 1-8, 2016.

[14] A. D. Winder, K. P. Maniar, J. J. Wei et al., "Synuclein- $\gamma$ in uterine serous carcinoma impacts survival: an NRG oncology/ gynecologic oncology group study: SNCG in uterine serous carcinoma," Cancer, vol. 13, no. 6, pp. 1144-1155, 2017.

[15] J. Zhao and N. Xing, "Identification of $\gamma$-synuclein as a stagespecific marker in bladder cancer by immunohistochemistry," Medical Science Monitor: International Medical Journal of Experimental and Clinical Research, vol. 20, no. 3, pp. 25502555, 2014.

[16] C. Liu, B. Shi, C. Hao et al., "Urine gamma-synuclein as a biomarker for the diagnosis of bladder cancer," Oncotarget, vol. 7, no. 2, pp. 43432-43441, 2016.

[17] M. M. Tomasiak-Lozowska, A. Bodzenta-Lukaszyk, M. Tomasiak, R. Skiepko, and Z. Zietkowski, "The role of interleukin 13 and interleukin 5 in asthma," Postępy Higieny $i$ Medycyny Doświadczalnej, vol. 64, no. 3, pp. 146-155, 2010.

[18] R. D. May and M. Fung, "Strategies targeting the IL-4/IL-13 axes in disease," Cytokine, vol. 75, no. 6, pp. 89-116, 2015.

[19] H. J. Kwon, J. E. Choi, and Y. K. Bae, "Interleukin-13 receptor alpha 2 expression in tumor cells is associated with reduced disease-free survival in patients with luminal subtype invasive breast cancer," Tumor Biology, vol. 40, no. 6, Article ID 101177, 2018.

[20] M. Terabe and J. A. Berzofsky, "Tissue-specific roles of NKT cells in tumor immunity," Frontiers in Immunology, vol. 9 , p. $1838,2018$.

[21] R. Peres, H. Furuya, I. Pagano, Y. Shimizu, K. Hokutan, and C. J. Rosser, "Angiogenin contributes to bladder cancer tumorigenesis by DNMT3b-mediated MMP2 activation," Oncotarget, vol. 7, no. 3, pp. 43109-43123, 2016.

[22] V. Urquidi, S. Goodison, J. Kim, M. Chang, Y. Dai, and C. J. Rosser, "Vascular endothelial growth factor, carbonic anhydrase 9, and angiogenin as urinary biomarkers for bladder cancer detection," Urology, vol. 79, no. 4, pp. 1185.e1-1185.e6, 2012.

[23] V. Verratti, L. Brunetti, R. Tenaglia et al., "Physiological analysis of 8-iso-PGF2 alpha: a homeostatic agent in superficial bladder cancer," Journal of Biological Regulators and Homeostatic Agents, vol. 25, no. 2, pp. 71-76, 2011.

[24] P. Mhawech-Fauceglia, L. Ali, R. T. Cheney et al., "Prognostic significance of neuron-associated protein expression in nonmuscle-invasive urothelial bladder cancer," Journal of Clinical Pathology, vol. 62, no. 8, pp. 710-714, 2009.

[25] K. MalekZadeh, M. Nikbakht, I. A. Sadeghi, S. K. Singh, and R. C. Sobti, "Overexpression of IL-13 in patients with bladder cancer," Cancer Investigation, vol. 28, no. 2, pp. 201-207, 2010.

[26] D. Margel, M. Pesvner-Fischer, J. Baniel, O. Yossepowitch, and I. R. Cohen, "Stress proteins and cytokines are urinary biomarkers for diagnosis and staging of bladder cancer," European Urology, vol. 59, no. 2, pp. 113-119, 2011.

[27] H. M. Mousa, A. N. Jassem, and M. J. Hussain, "The IHC expression of IL-13 in bladder tumour," in Proceedings of Special Second International Scientific Conference for the Life Sciences Faculty of Education for Women, pp. 117-119, Kufa, Iraq, 2018.

[28] N. Srabovic, Z. Mujagic, J. Mujanovic-Mustedanagic, Z. Muminovic, A. Softic, and L. Begic, "Interleukin 13 expression in the primary breast cancer tumor tissue," Biochemia Medica, vol. 21, no. 1, pp. 131-138, 2013.
[29] M. Babjuk, A. Böhle, M. Burger et al., "EAU guidelines on non-muscle-invasive urothelial carcinoma of the bladder: update 2016," European Urology, vol. 71, no. 3, pp. 447-461, 2017.

[30] Y. M. Ferro, G. Di Lorenzo, M. D. Vartolomei, D. Bruzzese, F. Cantiello, and G. Lucarelli, "Absolute basophil count is associated with time to recurrence in patients with high-grade T1 bladder cancer receiving bacillus Calmette-Guérin after transurethral resection of the bladder tumor," World Journal of Urology, vol. 38, no. 2, pp. 143-150, 2020.

[31] Y. Miyata, S. Kanda, K. Ohba et al., "Lymphangiogenesis and angiogenesis in bladder cancer: prognostic implications and regulation by vascular endothelial growth factors- $\mathrm{A},-\mathrm{C}$, and -D," Clinical Cancer Research, vol. 12, no. 3, pp. 800-806, 2006.

[32] S. Shirotake, A. Miyajima, T. Kosaka et al., "Angiotensin II type 1receptor expression and micro vessel density in human bladder cancer," Urology, vol. 77, no. 4, pp. 1009.e19-1009.e25, 2011.

[33] G. Shah, G. Zhang, F. Chen, Y. Cao, B. Kalyanaraman, and W. See, "iNOS expression and NO production contribute to the direct effects of BCG on urothelial carcinoma cell biology," Urologic Oncology, vol. 32, no. 1, pp. 45.e1-45.e9, 2014.

[34] G. Shah, J. Zielonka, F. Chen et al., " $\mathrm{H}_{2} \mathrm{O}_{2}$ generation by bacillus calmette-guérin induces the cellular oxidative stress response required for bacillus calmette-guérin direct effects on urothelial carcinoma biology," Journal of Urology, vol. 192, no. 4, pp. 1238-1248, 2014.

[35] M. See, T. Fleming, M. Robinson et al., "Global, regional, and national prevalence of overweight and obesity in children and adults during 1980-2013: a systematic analysis for the global burden of disease study 2013," The Lancet, vol. 384, no. 9945, pp. 766-781, 2014.

[36] M. Ferro, M. Katalin, C. Buonerba et al., "Type 2 diabetes mellitus predicts worse outcomes in patients with high-grade T1 bladder cancer receiving bacillus Calmette-Guerin after transurethral resection of the bladder tumor," Urologic Oncology: Seminars and Original Investigations, vol. 38, no. 2, pp. 459-464, 2020.

[37] G. De Pergola and F. Silvestris, "Obesity as a major risk factor for cancer," International Journal of Obesity, vol. 201311 pages, Article ID 291546, 2013.

[38] M. Ferro, M. D. Vartolomei, G. I. Russo, F. Cantiello, A. Rahman, and A. Farhan, "An increased body mass index is associated with a worse prognosis in patients administered BCG immunotherapy for T1 bladder cancer," World Journal of Urology, vol. 37, no. 2, pp. 507-514, 2019.

[39] J. C. Pardo, V. Ruiz de Porras, A. Plaja et al., "Moving towards personalized medicine in muscle-invasive bladder cancer: where are we now and where are we going?" International Journal of Molecular Sciences, vol. 21, no. 17, p. 6271, 2020. 